\title{
Editorial: Synthesis, Characterization, and Applications of Magneto-Responsive Functional Materials
}

\begin{abstract}
Yancheng $\mathrm{Li}^{1 *}$, Xinglong Gong ${ }^{2}$, Yingdan $\mathrm{Liu}^{3}$ and Jinbo $W u^{4}$
${ }^{1}$ School of Civil and Environmental Engineering, University of Technology Sydney, Sydney, NSW, Australia, ${ }^{2}$ CAS Key Laboratory of Mechanical Behavior and Design of Materials, Department of Modern Mechanics, CAS Center for Excellence in Complex System Mechanics, University of Science and Technology of China (USTC), Hefei, China, ${ }^{3}$ State Key Lab of Metastable Materials Science and Technology, College of Materials Science and Engineering, Yanshan University, Qinhuangdao, China, ${ }^{4}$ Materials Genome Institute, Shanghai University, Shanghai, China
\end{abstract}

Keywords: magneto-responsive, magnetorheological, characterization, application, numerical

Editorial on the Research Topic

Synthesis, Characterization, and Applications of Magneto-Responsive Functional Materials

\section{BACKGROUND}

Magneto-responsive functional materials, in particular, magnetorheological (MR) materials (in forms of liquid, elastomer, grease, or others), have gained significant attention from around the world. The materials, with drastic rheological property changes, have been heavily investigated to develop smart devices and applications, ranging from micro-sized sensors to large-scale energy dissipation devices for civil engineering applications. Cross-disciplinary research activities are still booming with aims to: 1) render larger MR effect with better stability and durability; 2) understand and characterize the complex material behavior with existing or new rheology theories; 3 ) apply innovations to design MR devices or actuators in many forms for respective targeted applications; and, 4) address underlying concerns from end-customers for application-related obstacles. This Research Topic is set to provide a platform for scientists and researchers to exchange the latest developments in the field. The topic includes wide coverage of the focusing theme on technologies and innovations in the aforementioned four areas. We are proud to present 10 peer-reviewed contributions offering insights on synthesis, characterization, and applications of MR materials from leading researchers.

\section{MAJOR HIGHLIGHTS OF THE CONTRIBUTIONS}

MR fluids as important MR material that are used to design various intelligent vibration control devices, such as brakes, clutches, dampers, and mounts, as well as medical apparatus. Over the past 2 decades, numerous designs have been proposed and tested based on the four operational modes of the MR fluid. To summarise the latest developments, Hua et al. presented a dedicated review of the structural configurations of MR fluid based devices in 2018-2020. In particular, the section on MR fluid devices for medical application includes several innovative devices, such as robotic bone biopsy, haptic actuator, and tactile device, which are highlights worthy of attention. Conclusive remarks and future perspectives are also offered by the authors. Another topic review on the role of MR fluid in 
precision machining is given by $\mathrm{Lu}$ et al. The state-of-the-art review on the surface polishing equipment is the first piece in the community to comprehend outstanding research and work in the area.

There has been a continuous effort in upgrading the performance of MR fluid, in terms of larger MR effect, low sedimentation, and high stability. When subjected to a magnetic field, the particles in the carrier form chain or column structures along the field direction, which can only be broken until an external force/stress reaches a certain threshold, namely yield stress. The improvement of MR effect can be achieved through modifications to the magneto particles and carrier fluids. The interface between particles and carrier fluids plays a substantial role in the performance of MR fluid. Tong et al. proposed the use of ionic liquid and silicon oil with the same viscosity as a carrier fluid to prepare MR fluid. They showed that the new MR fluid possesses higher yield stress of up to $90 \mathrm{kPa}$, around $20 \mathrm{kPa}$ more than a silicon oil based MR fluid. The results of this experiment are explained by the enhanced interactions between the particles, encouraged by the fact that ion fragments in the ionic liquid formed an ion layer on the surface of the particles. Jang et al. adopted another approach to enhance the MR effect, i.e., coating Carbon Iron (CI) particles with hard-magnetic barium ferrite (BF) nanoparticles. The hard-magnetic BF nanoparticles have a hexagonal plate-like structure and high magnetocrystalline anisotropy, high Curie point, relatively high magnetic saturation value and coercive force, and superior chemical stability and corrosion resistance. SEM images reveal that the $\mathrm{BaFe}_{12} \mathrm{O}_{19}$ particles are attached to the space between the CI particles, which enhanced the MR efficiency, reduced particle aggregation, and thus improved suspension stability.

The gravitational force of the heavy ferrite particle induces settling of the particles due to the mismatch with the low-density carrier fluid. Sedimentation in MR fluid has indeed become a burning issue to resolve facing the application of MR devices, or at least with technology to monitor the sedimentation inside the device. Zhang et al. propose an in-situ capacitance sensing approach to capture the settlement of MR fluid within an MR damper cylinder. They designed an open plate capacitor configuration and the system was calibrated using theoretical simulation and experiment. The proposed system can be used for the long-term monitoring of the particle settling of MR fluid in any fabricated MR device.

To overcome the influence of disturbances, either internal or external, on the performance of the MR impact buffer system, B. Wang and Li. proposed a hybrid control strategy with sliding mode active disturbance rejection control based on extended state observer. The proposed control method is validated through numerical simulation and exhibits superiority with fast response, minimal overshooting, and great immunity to nonlinear hysteresis.

Besides MR fluids, other forms of MR materials such as MR elastomer, gel, and grease, have been been the focus of recent research. Similar to the trajectory of MR fluids, research activities on other MR materials are mainly from material scientists and are expected to rapidly shift to engineering. Wang et al. explored the feasibility of MR elastomer (also called Magneto-Sensitive rubber, or MS rubber) to be used in semi-active vehicle suspension. A constitutive model was proposed to accurately replicate the behavior of MS rubber. They then used an $\mathrm{H}$-infinity control strategy for a quarter car model featuring MS rubber semi-active suspension to examine the performances under a bump and a random ground excitation. Future perspectives on this proposal are discussed, with ample insights presented. Gong et al. developed a novel MR polymer gel utilizing carbon nanotubes and CI particles to mix into the polymer gel matrix. An unusual nonlinear magneto-electro-mechanical response, i.e. ratedependence, was observed together with high performance sensing behavior. Thus, the new material has promising futures in both active control and electromechanical sensing, either separately or combined. Mao et al. investigated the magneto-induced normal stress in MR gel under quasistatically monotonic and periodically cyclic loading. Detailed experimental testing and observation can all be found in this article with ample insights.

Understanding the rheological properties is the key to unlock the potentials of MR materials for future applications. Usually, storage/loss modules and Lissajous curves from the oscillatory shear tests are used to extrapolate the rheological properties. However, they each possess drawbacks in making the detailed nonlinear behavior more explicit. Wang et al. utilized Fourier transform-Chebyshev analysis to quantitatively interpret the influence and frequency on the non-linear rheology of MR grease. The strain-stiffening/softening and shear thickening/ thinning features of the materials can be easily expressed using this method, which offers a new way of examining the complex behavior of MR materials, especially under sophisticated influences of the magnetic field, complex strain, and temperature.

\section{SUMMARY}

These Research Topic contributions provide information about the latest developments in magneto-responsive materials in relation to various aspects, such as state-of-the-art review, material development, and characterization, constitutive modelling, numerical methods, and simulation. The editors hope that you find these articles useful and are inspired by them.

\section{AUTHOR CONTRIBUTIONS}

\section{YL prepared the draft. XG, YdL, and JW edited the draft.}

Conflict of Interest: The authors declare that the research was conducted in the absence of any commercial or financial relationships that could be construed as a potential conflict of interest.

Copyright (C) $2021 \mathrm{Li}$, Gong, Liu and Wu. This is an open-access article distributed under the terms of the Creative Commons Attribution License (CC BY). The use, distribution or reproduction in other forums is permitted, provided the original author(s) and the copyright owner(s) are credited and that the original publication in this journal is cited, in accordance with accepted academic practice. No use, distribution or reproduction is permitted which does not comply with these terms. 